\title{
Composition hyperrings
}

\author{
Irina Cristea and Sanja Jančić-Rašović
}

\begin{abstract}
In this paper we introduce the notion of composition hyperring. We show that the composition structure of a composition hyperring is determined by a class of its strong multiendomorphisms. Finally, the three isomorphism theorems of ring theory are derived in the context of composition hyperrings.
\end{abstract}

\section{Introduction}

The hyperrings have appeared as a new class of algebraic hyperstructures more general than that of hyperfields, introduced by Krasner [9] in the theory of valued fields. A Krasner hyperring is a nonempty set $R$ endowed with a hyperoperation (the addition) and a binary operation (the multiplication) such that $(R,+)$ is a canonical hypergroup, $(R, \cdot)$ is a semigroup and the multiplication is distributive with respect to the addition. The theory of these hyperrings has been developing since the beginning of seventies, thanks to the contributions of Mittas [14, 15], Krasner [10], Stratigopoulos [20], till nowadays $[2,3,5,8,13,17]$.

Several types of hyperrings have been proposed (for more details see $[6,11$, $12,16,22]$ and their references), but the most general one is that introduced by Spartalis [18], used also in the context of $P$-hyperrings or $(H, R)$-hyperrings [19]. A comprehensive review of hyperrings theory is covered in Nakassis [16], Vougiouklis [22] and in the book [6] written by Davvaz, Leoreanu-Fotea. New applications of the theory of hyperrings in number theory and algebraic geometry can be found in $[4,21]$.

Key Words: Hyperring; Hyperideal; Multiendomorphism; Composition hyperoperation. 2010 Mathematics Subject Classification: Primary 20N20; Secondary 06E20.

Received: May 2012.

Accepted: June 2012. 
Based on the notion of composition ring introduced by Adler [1], we define here the concept of composition hyperring, as a quadruple $(R,+, \cdot, \circ)$ such that $(R,+, \cdot)$ is a commutative hyperring in the general sense of Spartalis, and the composition hyperoperation $\circ$ is an associative hyperoperation, distributive to the right side with respect to the addition and multiplication. Many of the familiar rings of functions are composition rings, where the composition operation is defined just as the composition between the functions. The idea to study a similar hyperstructure comes from the properties of the operations between the polynomials with coefficients in a commutative hyperring, the set of them forming a hyperring as shown in [7] by Jančić-Rašović.

The rest of the paper is organized as follows. After a short presentation of the main results from hyperring theory covered in Preliminaries, in Section 3, we define the notion of composition hyperring, proving that the composition structure of it is determined by a certain class $\left(\phi_{y}\right)_{y \in R}$ of strong multiendomorphisms of the considered hyperring $R$. We determine conditions under which an arbitrary family $\Omega$ of multiendomorphims of $R$ generates the class $\left(\phi_{y}\right)_{y \in R}$ of strong multiendomorphisms of $R$, and, consequently, the composition hy-

peroperation on $R$. In Section 4, using the notion of composition hyperideal of a composition hyperring, the three isomorphism theorems of ring theory are derived and discussed in the context of composition hyperrings. We end this note with some concluding remarks and some open problems.

\section{Preliminaries}

We recall some definitions concerning hyperrings theory and we fix the notations used in this paper.

A canonical hypergroup is a nonempty set $H$ endowed with an additive hyperoperation $+: H \times H \longrightarrow \mathcal{P}^{*}(H)$, satisfying the following properties:

1. for any $x, y, z \in H, x+(y+z)=(x+y)+z$

2. for any $x, y \in H, x+y=y+x$

3. there exists $0 \in H$ such that $0+x=x+0=x$, for any $x \in H$

4. for every $x \in H$, there exists a unique element $x^{\prime} \in H$, such that $0 \in$ $x+x^{\prime}$ (we write $-x$ instead of $x^{\prime}$ and we call it the opposite of $x$.)

5. $z \in x+y$ implies that $y \in-x+z$ and $x \in z-y$, that is $(H,+)$ is reversible.

A multivalued system $(R,+, \cdot)$ is a hyperring (in the general case of Spartalis [18]), if: 
1. $(R,+)$ is a hypergroup

2. $(R, \cdot)$ is a semihypergroup

3. the multiplication is distributive with respect to the addition, i.e. for all $x, y, z \in R, x \cdot(y+z)=x \cdot y+x \cdot z$ and $(x+y) \cdot z=x \cdot z+y \cdot z$.

If $R$ is commutative with respect to both addition and multiplication, then it is called a commutative hyperring.

A particular case of hyperring is that called Krasner hyperring, where $(R,+)$ is a canonical hypergroup, $(R, \cdot)$ is a semigroup having 0 as a bilaterally absorbing element, and the multiplication is distributive with respect to the addition.

A nonempty subset $S$ of a hyperring $R$ is called a subhyperring of $R$ if, $(S,+)$ is a subhypergroup of $(R,+)$ and $S \cdot S \subseteq S$. Moreover, a subhyperring $S$ of a hyperring $R$ is a hyperideal of $R$, if $r \cdot x \subseteq S$ and $x \cdot r \subseteq S$, for all $r \in R$ and $x \in S$.

Suppose now that $(R,+, \cdot)$ and $\left(T,+^{\prime},,^{\prime}\right)$ are two hyperrings. A map $\phi$ : $R \longrightarrow T$ is called a multihomomorphism from $R$ to $T$ if, for all $x, y \in R$, the following relations hold:

1. $\bigcup_{u \in x+y} \phi(u) \subseteq \phi(x)+^{\prime} \phi(y)$

2. $\bigcup_{u \in x \cdot y} \phi(u) \subseteq \phi(x) \cdot^{\prime} \phi(y)$

If, in the previous conditions, the equality is valid, then $\phi$ is called a strong multihomomorphism. A multihomomorphism from $R$ to $R$ is called multiendomorphism of $R$. If $\phi_{1}$ and $\phi_{2}$ are multiendomorphisms on a hyperring $R$, then their composition $\phi_{1} \circ \phi_{2}$ defined by $\left(\phi_{1} \circ \phi_{2}\right)(x)=\bigcup_{a \in \phi_{2}(x)} \phi_{1}(a)$ is also a multiendomorphism on $R$.

\section{Composition hyperrings}

In this section we introduce the notion of composition hyperring, giving several examples that illustrate the significance of this new hyperstructure. The composition rings constructed by Adler [1] represents a special case of composition hyperrings.

A composition ring is a commutative ring $R$ with an additional binary operation $\circ$ (called composition), satisfying the following properties:

1. $(x+y) \circ z=x \circ z+y \circ z$

2. $(x y) \circ z=(x \circ z)(y \circ z)$

3. $x \circ(y \circ z)=(x \circ y) \circ z$, 
for any $x, y, z \in R$. The most significant and natural example of a such ring is represented by the ring of functions, where the composition operation is just the composition between two functions: $(f \circ g)(x)=f(g(x))$. Extending this construction to the case of hyperstructures, we obtain the following concept.

Definition 3.1. A composition hyperring is an algebraic structure $(R,+, \cdot, \circ)$, where $(R,+, \cdot)$ is a commutative hyperring and the hyperoperation $\circ$ satisfies the following properties, for any $x, y, z \in R$ :

1. $(x+y) \circ z=x \circ z+y \circ z$

2. $(x \cdot y) \circ z=(x \circ z) \cdot(y \circ z)$

3. $x \circ(y \circ z)=(x \circ y) \circ z$.

The binary hyperoperation $\circ$ having the previous properties is called the composition hyperoperation of the hyperring $(R,+, \cdot)$.

Definition 3.2. Let $(R,+, \cdot, \circ)$ be a composition hyperring. An element $c \in R$ is called a constant, if $c \circ x=c$, for all $x \in R$. If $A$ is an arbitrary subset of $R$, the set of all constants in $A$ is called a foundation of $A$, denoted by FoundA.

Example 3.3. Let $(R,+, \cdot)$ be a commutative hyperring. A formal power series with coefficients in $R$ is an infinite sequence $\left(a_{0}, a_{1}, \ldots, a_{n}, \ldots\right)$ in which all $a_{i}$ belong to $R$. The set of all formal power series with coefficients in $R$ will be denoted by $R[[x]]$. Defining the following hyperoperations $\oplus$ and $\odot$ by taking:

$$
\left(a_{0}, a_{1}, \ldots, a_{n}, \ldots\right) \oplus\left(b_{0}, b_{1}, \ldots, b_{n}, \ldots\right)=\left\{\left(c_{0}, c_{1}, \ldots, c_{n}, \ldots\right) \mid c_{k} \in a_{k}+b_{k}\right\}
$$

and

$$
\left(a_{0}, a_{1}, \ldots, a_{n}, \ldots\right) \odot\left(b_{0}, b_{1}, \ldots, b_{n}, \ldots\right)=\left\{\left(c_{0}, c_{1}, \ldots, c_{n}, \ldots\right) \mid c_{k} \in \sum_{i+j=k} a_{i} b_{j}\right\}
$$

then the obtained hyperstructure $(R[[x]], \oplus, \odot)$ is a hyperring.

Suppose now that the hypergroup $(R,+)$ has one identity, the zero element 0 . Let $R[x]$ denote the set of all polynomials $\left(a_{0}, a_{1}, \ldots, a_{n}, \ldots\right)$ of $R[[x]]$ having $a_{i}=0$ except a finite number of indices $i$. If $0+0=0$ and $a \cdot 0=0$, for all $a \in R$, then according to Theorem 3.2. [7], it follows that $(R[x], \oplus, \odot)$ is a subhyperring of $(R[[x]], \oplus, \odot)$.

Take $f=\left(a_{0}, a_{1}, \ldots, a_{n}, \ldots\right) \in R[x]$ such that $a_{k}=0$, for all $k \geq n+1$, and take $g \in R[x]$. Define a new hyperoperation by putting:

$$
f \circ g=a_{0} \oplus\left(a_{1} \odot g\right) \oplus \ldots \oplus\left(a_{n} \odot g^{n}\right) .
$$

It can be easily verified that $(R[x], \oplus, \odot, \circ)$ is a composition hyperring with Found $(R)=R$. 
Example 3.4. If $(R,+, \cdot)$ is an arbitrary commutative hyperring and $\circ$ is defined by $r \circ s=r$, for all $r, s \in R$, then $(R,+, \cdot, \circ)$ is a composition hyperring with $\operatorname{Found}(R)=R$.

Example 3.5. Let $(R,+, \cdot)$ be a commutative ring and $R^{R}$ be the ring of all functions from $R$ into $R$. If we define the binary operation $\circ$ as the composition of functions, then $\left(R^{R},+, \cdot, \circ\right)$ becomes a composition ring with $\operatorname{Found}(R)=$ $R$.

In the following we propose a method to define the composition structure of a composition hyperring by a certain class of its strong multiendomorphisms.

Theorem 3.6. Let $(R,+, \cdot, \circ)$ be a composition hyperring. For any element $y \in R$, the function $\Phi_{y}: R \longrightarrow \mathcal{P}^{*}(R)$ defined by $\Phi_{y}(x)=x \circ y$, for all $x \in R$, is a strong multiendomorphism of the hyperring $R$. Moreover, if $M$ is a nonempty subset of $R$, denote by

$$
\Phi_{M}(x)=\bigcup_{m \in M} \Phi_{m}(x), \forall x \in R .
$$

Then, for all $x, y, z \in R$, it holds:

$$
\Phi_{\Phi_{x}(y)}(z)=\bigcup_{t \in \Phi_{y}(z)} \Phi_{x}(t) .
$$

Conversely, if $(R,+, \cdot)$ is a commutative hyperring and $\left(\Phi_{y}\right)_{y \in R}$ is a family of its strong multiendomorphisms satisfying equation (1), then, defining the hyperoperation $\circ$ by $x \circ y=\Phi_{y}(x)$, we obtain that $(R,+, \cdot, \circ)$ is a composition hyperring.

Proof. Let $(R,+, \cdot, \circ)$ be a composition hyperring and let $y \in R$. By the definition of the function $\Phi_{y}$, for all $a, b \in R$, it holds:

$$
\bigcup_{u \in a+b} \Phi_{y}(u)=\bigcup_{u \in a+b} u \circ y=(a+b) \circ y=a \circ y+b \circ y=\Phi_{y}(a)+\Phi_{y}(b)
$$

and

$$
\bigcup_{u \in a \cdot b} \Phi_{y}(u)=\bigcup_{u \in a \cdot b} u \circ y=(a \cdot b) \circ y=(a \circ y) \cdot(b \circ y)=\Phi_{y}(a) \cdot \Phi_{y}(b) .
$$

Thus, $\Phi_{y}$ is a strong multiendomorphism of the hyperring $(R,+, \cdot)$. Moreover, for all $x, y, z \in R$, it holds:

$$
\begin{aligned}
\Phi_{\Phi_{x}(y)}(z) & =\bigcup_{s \in y \circ x} \Phi_{s}(z)=\bigcup_{s \in y \circ x} z \circ s=z \circ(y \circ x)=(z \circ y) \circ x= \\
& =\bigcup_{t \in z \circ y} t \circ x=\bigcup_{t \in \Phi_{y}(z)} \Phi_{x}(t) .
\end{aligned}
$$


Suppose now that $\left(\Phi_{y}\right)_{y \in R}$ is a family of strong multiendomorphisms of a hyperring $(R,+, \cdot)$ satisfying condition (1). If a hyperoperation $\circ$ is defined taking $x \circ y=\Phi_{y}(x)$, then it can be easily verified that, for all $a, b, x \in R$, it holds $(a+b) \circ x=a \circ x+b \circ x$ and $(a \cdot b) \circ x=(a \circ x) \cdot(b \circ x)$.

Besides, using equation (1), we obtain

$$
\begin{aligned}
(a \circ b) \circ c & =\bigcup_{s \in \Phi_{b}(a)} s \circ c=\bigcup_{s \in \Phi_{b}(a)} \Phi_{c}(s)=\Phi_{\Phi_{c}(b)}(a) \\
& =\bigcup_{u \in \Phi_{c}(b)} \Phi_{u}(a)=\bigcup_{u \in b \circ c} a \circ u=a \circ(b \circ c) .
\end{aligned}
$$

Thus, $(R,+, \cdot, \circ)$ is a composition hyperring and the proof is now complete.

It arises the following question: Can every commutative hyperring give a composition structure? In this proposal we determine conditions under which a family of multiendomorphisms of a hyperring $(R,+, \cdot)$ generates the class $\left(\Phi_{y}\right)_{y \in R}$ satisfying the conditions of the previous theorem.

Let $\Omega$ be a family of multiendomorphisms of a hyperring $(R,+, \cdot)$. For any $y \in R$, denote

$$
P_{y}=\bigcup_{\Phi \in \Omega} \Phi(y) .
$$

The set $P_{y}$ is called the orbit of $y$. An orbit $P$ is said to be principal if, for all $x \in P$ and $\Phi_{1}, \Phi_{2} \in \Omega$, it holds:

$$
\Phi_{1}(x) \cap \Phi_{2}(x) \neq \emptyset \Longrightarrow \Phi_{1}=\Phi_{2} .
$$

Let $(R,+, \cdot)$ be a commutative hyperring and 0 be an identity element of the hypergroup $(R,+, \cdot)$.

Lemma 3.7. Let $\Omega$ be a family of strong multiendomorphisms of a hyperring $(R,+, \cdot)$, such that:

1. $\Phi_{1} \circ \Phi_{2} \in \Omega$, for all $\Phi_{1}, \Phi_{2} \in \Omega$, where $\Phi_{1} \circ \Phi_{2}$ is defined by: $\left(\Phi_{1} \circ \Phi_{2}\right)(x)=$ $\bigcup_{v \in \Phi_{2}(x)} \Phi_{1}(v)$.

2. $\Phi(0)=0$

3. For all $x, y \in R$ it holds:

$$
\Phi \in \Omega \text { and } x \in \Phi(y) \Longrightarrow \exists \Phi_{1} \in \Omega \text { such that } y \in \Phi_{1}(x)
$$

Then, $\Omega$ induces a partition of the set $\Omega(R)=\bigcup_{\Phi \in \Omega, r \in R} \Phi(r)$ into orbits. 
Proof. It is clear that $\Omega(R)=\bigcup_{y \in R} P_{y}$.

First we prove that $x \in P_{y}$ implies that $P_{x}=P_{y}$. Indeed, if $x \in P_{y}$, then $x \in \Phi(y)$, for some $\Phi \in \Omega$. By the third condition of the hypothesis, it follows that $y \in \Phi_{1}(x)$, for some $\Phi_{1} \in \Omega$. Thus, if $z \in P_{y}$, then there exists $\Phi_{2} \in \Omega$ such that $z \in \Phi_{2}(y)$ and so $z \in\left(\Phi_{2} \circ \Phi_{1}\right)(x)$. Since $\Phi_{2} \circ \Phi_{1} \in \Omega$, by the first condition of the hypothesis, we obtain $z \in P_{x}$. Therefore, $x \in P_{y}$ implies $P_{y} \subseteq P_{x}$. Moreover, if $x \in P_{y}$, then $y \in P_{x}$ and consequently $P_{x} \subseteq P_{y}$, that is $P_{x}=P_{y}$.

Thus if $P_{x} \cap P_{y} \neq \emptyset$, then there exists $z \in P_{x} \cap P_{y}$ which implies that $P_{x}=P_{y}=P_{z}$ as we have proved before. Thereby, $\Omega(R)$ can be partitioned into the orbits $P_{y}, y \in R$.

Notice that, if the family $\Omega$ satisfies the three conditions of the previous lemma and if $\Omega$ has at least two elements, then, for any principal orbit $P$, it holds $0 \notin P$.

Theorem 3.8. Let $\Omega$ be a family of strong multiendomorphisms of a hyperring $(R,+, \cdot)$ satisfying conditions of Lemma 3.7. Let $\mathcal{S}$ be a nonempty set of principal orbits with $0 \notin \mathcal{S}$ and for each $P \in \mathcal{S}$, let $a_{p}$ be an element of $P$.

For each $y \in R$ define the multiendomorphism $\Phi_{y}: R \longrightarrow \mathcal{P}^{*}(R)$ as follows. If $y$ is an element of an orbit $P \in \mathcal{S}$, then $\Phi_{y}=\Phi$, where $\Phi$ is an element of $\Omega$ such that $y \in \Phi\left(a_{p}\right)$. If $y \notin \bigcup_{P \in \mathcal{S}} P$, then $\Phi_{y}=0$.

Then, the family $\left(\Phi_{y}\right)_{y \in R}$ satisfies equation (1), thus it generates a composition hyperoperation on $R$.

Proof. If $P_{1}, P_{2} \in \mathcal{S}$ and $y \in P_{1} \cap P_{2}$, then, by Lemma 3.7, it follows that $P_{1}=P_{2}$. Besides, if $y \in P$ and $y \in \Phi_{1}\left(a_{p}\right) \cap \Phi_{2}\left(a_{p}\right)$, then $\Phi_{1}=\Phi_{2}$, because $P$ is a principal orbit. So the mapping $y \longrightarrow \Phi_{y}$ is well defined.

Obviously, $\left(\Phi_{y}\right)_{y \in R}$ is a family of strong multiendomorphisms of $R$.

Let $x, y, a \in R$. We will prove the following relation:

$$
\Phi_{\Phi_{x}(y)}(a)=\bigcup_{v \in \Phi_{y}(a)} \Phi_{x}(v) .
$$

We have to consider the following situations.

1) If $x \in \bigcup_{P \in \mathcal{S}} P$, then $\Phi_{x}=\Phi$, where $\Phi$ is an element of $\Omega$ such that $x \in \Phi\left(a_{p}\right)$, with $x \in P$. We have two possibilities:

a) If $y \in P^{\prime}$, for some $P^{\prime} \in \mathcal{S}$, then $\Phi_{y}=\Phi^{\prime}$, where $\Phi^{\prime}$ is an element of $\Omega$ such that $y \in \Phi^{\prime}\left(a_{p^{\prime}}\right)$. Thus, $\Phi_{x}(y)=\Phi(y) \subseteq\left(\Phi \circ \Phi^{\prime}\right)\left(a_{p^{\prime}}\right)$. Therefore, if $z \in \Phi_{x}(y)$, then $z \in\left(\Phi \circ \Phi^{\prime}\right)\left(a_{p^{\prime}}\right)$. Since $\Phi \circ \Phi^{\prime} \in \Omega$, it follows that $z \in P^{\prime}$ and $\Phi_{z}=\Phi \circ \Phi^{\prime}$. Thus, $\bigcup_{z \in \Phi_{x}(y)} \Phi_{z}(a)=\left(\Phi \circ \Phi^{\prime}\right)(a)=\left(\Phi_{x} \circ \Phi_{y}\right)(a)$. So, the equation (2) holds. 
b) Suppose $y \notin \bigcup_{P \in \mathcal{S}} P$. Then $\Phi_{x}(y) \cap \bigcup_{P \in \mathcal{S}} P=\emptyset$. Indeed, if $z \in$ $\Phi_{x}(y) \cap P^{\prime}$, for some $P^{\prime} \in \mathcal{S}$, then $z \in \Phi_{x}(y)=\Phi(y)$ and so there exists $\Phi_{1} \in \Omega$ such that $y \in \Phi_{1}(z)$. Since $z \in P^{\prime}$, there exists $\Phi_{2} \in \Omega$ such that $z \in \Phi_{2}\left(a_{p^{\prime}}\right)$. Therefore, $y \in\left(\Phi_{1} \circ \Phi_{2}\right)\left(a_{p^{\prime}}\right)$, and because $\Phi_{1} \circ \Phi_{2} \in \Omega$, we obtain $y \in P^{\prime}$, which contradicts the assumption.

Therefore, if $y \notin \bigcup_{P \in \mathcal{S}} P$, then, for all $z \in \Phi_{x}(y)$, it holds $\Phi_{z}=0$ and so $\Phi_{\Phi_{x}(y)}(a)=0$. Also, $\bigcup_{v \in \Phi_{y}(a)} \Phi_{x}(v)=\Phi_{x}(0)=0$.

2) Suppose now $x \notin \bigcup_{P \in \mathcal{S}} P$. Then $\Phi_{x}=0$ and so $\Phi_{x}(y)=0$. Thus, $\Phi_{\Phi_{x}(y)}(a)=\Phi_{0}(a)$.

Notice that $0 \notin \bigcup_{P \in \mathcal{S}} P$. Indeed, if $0 \in P$, for some $P \in \mathcal{S}$, then, by Lemma 3.7, it follows that $P=P_{0}=\bigcup_{\Phi \in \Omega} \Phi(0)=0$, i.e. we obtain $0 \in \mathcal{S}$, contrary to the hypothesis. Thus, $0 \notin \bigcup_{P \in \mathcal{S}} P$ and so $\Phi_{0}=0$, i.e. $\Phi_{0}(a)=$ 0 . So, $\Phi_{\Phi_{x}(y)}(a)=0$. Also $\bigcup_{v \in \Phi_{y}(a)} \Phi_{x}(v)=0$, since $\Phi_{x}=0$. Thus, the family $\left(\Phi_{y}\right)_{y \in R}$ satisfies conditions of Theorem 3.6, generating a composition hyperoperation on $R$.

Remark 3.9. Let $(R,+, \cdot)$ be a Krasner hyperring and Aut $R$ be the group of its ordinary automorphisms. If $\Omega$ is a subgroup of Aut $R$, then $\Omega$ satisfies conditions of previous theorem. The composition hyperoperation $\circ$ associated with the corresponding family $\left(\Phi_{y}\right)_{y \in R}$ is an ordinary operation, since, for all $x, y \in R,|x \circ y|=\left|\Phi_{y}(x)\right|=1$.

Example 3.10. Let $(R,+, \cdot)$ be the field $\mathbb{R}$ of real numbers and $A=2^{\mathbb{Q}}=$ $\left\{2^{q} \mid q \in \mathbb{Q}\right\}$. Define hyperoperations $\oplus_{A}$ and $\odot_{A}$ on $R$ by: $x \oplus_{A} y=x A+y A$ and $x \odot_{A} y=x A y$. It can be easily verified that $\left(R, \oplus_{A}\right)$ is a commutative hypergroup and $\left(R, \odot_{A}\right)$ is a commutative semihypergroup. Moreover, since, for all $a \in A$, it holds $a A=A$, it follows that:

$$
\begin{aligned}
\left(x \oplus_{A} y\right) \odot_{A} z & =(x A+y A) A z=(x A z+y A z) A=(x z A+y z A) A= \\
& =\bigcup_{a \in A}(x z A a+y z A a)=x z A+y z A=x z A A+y z A A= \\
& =\left(x \odot_{A} z\right) \oplus_{A}\left(y \odot_{A} z\right),
\end{aligned}
$$

for all $x, y, z \in R$. Thus, $\left(R, \oplus_{A}, \odot_{A}\right)$ is a commutative hyperring.

Let us define now two functions $f: R \longrightarrow \mathcal{P}^{*}(R)$ and $g: R \longrightarrow \mathcal{P}^{*}(R)$ by $f(x)=A \cdot x=\left\{2^{q} \cdot x \mid q \in \mathbb{Q}\right\}$ and $g(x)=-A \cdot x=\left\{-2^{q} \cdot x \mid q \in \mathbb{Q}\right\}$. Obviously, $f$ and $g$ are strong multiendomorphisms of $\left(R, \oplus_{A}, \odot_{A}\right)$. Also, $f \circ f=g \circ g=f$ and $f \circ g=g \circ f=g$. If $x \in f(y)$, then $x=2^{q} y$, for some $q \in \mathbb{Q}$, and so $y=2^{-q} x \in A x=f(x)$. Similarly, $x \in g(y)$ implies that $y \in g(x)$. Obviously $f(0)=0$ and $g(0)=0$. Let $\Omega=\{f, g\}$. It is easy to verify that $\Omega$ satisfies conditions of Lemma 3.7. 
Besides, for any $y \in R$, its orbit has the form $P_{y}=f(y) \cup g(y)=\left\{ \pm 2^{q} \cdot y \mid\right.$ $q \in \mathbb{Q}\}$.

If $y \neq 0$, then $P_{y}$ is a principal orbit, since, for any $x \in P_{y}$, it holds $f(x) \cap g(x)=\emptyset$, because $2^{\mathbb{Q}} x \cap\left(-2^{\mathbb{Q}} x\right)=\emptyset$. Thus, by the previous theorem, each family $\mathcal{S}$ of principal orbits generates corresponding composition hyperoperation on $R$.

For instance, if $\mathcal{S}=\left\{P_{n} \mid n \in \mathbb{N}\right\}$, then, for $y \in \bigcup_{n \in \mathbb{N}} P_{n}$ and $y>0$, we put $\Phi_{y}=f$ and, for $y<0$, we put $\Phi_{y}=g$. If $y \notin \bigcup_{n \in \mathbb{N}} P_{n}$, then $\Phi_{y}=0$. Thus, the corresponding hyperoperation is defined by:

$$
x \circ y= \begin{cases}2^{\mathbb{Q}} x & \text { if } y \in 2^{\mathbb{Q}} \cdot \mathbb{N}, \\ -2^{\mathbb{Q}} x & \text { if } y \in-2^{\mathbb{Q}} \cdot \mathbb{N}, \\ 0 & \text { otherwise. }\end{cases}
$$

\section{Isomorphism theorems of composition hyperrings}

This section deals with the isomorphism theorems for the composition hyperrings. In order to state them, first we introduce the notion of composition hyperideal and then we construct the quotient composition hyperring.

Throughout this section, $(R,+, \cdot, \circ)$ is a composition hyperring such that $(R,+)$ is a canonical hypergroup and $x \cdot 0=0$, for all $x \in R$. Obviously, in a Krasner hyperring these conditions are satisfied.

Definition 4.1. Let $(R,+, \cdot, \circ)$ be a composition hyperring and $N$ be a subset of $R$. $N$ is called a composition hyperideal of $R$ if the following three conditions are satisfied:

1. $N$ is a hyperideal of the hyperring $R$

2. $n \circ r \subseteq N$, for all $n \in N$ and $r \in R$

3. If $r, s, t \in R$ and $r-s \cap N \neq \emptyset$, then $t \circ r-t \circ s \subseteq N$.

Let $N$ be a composition hyperideal of $R$. Consider on $R$ the following relation:

$$
x \rho y \Longleftrightarrow x+N=y+N .
$$

Obviously, $\rho$ is an equivalence on $R$ and the equivalence class represented by $x$ is $[x]_{\rho}=x+N$. Denote by $R / N$ the set of all equivalence classes of the elements of $R$ with respect to the equivalence relation $\rho$.

Lemma 4.2. Let $R$ be a composition hyperring and $N$ be a composition hyperideal of $R$. Defining on the quotient $R / N$ the hyperoperations $\oplus, \odot, \odot$ as it 
follows:

$$
\begin{aligned}
& (x+N) \oplus(y+N)=\{z+N \mid z \in x+y\} \\
& (x+N) \odot(y+N)=\{z+N \mid z \in x \cdot y\} \\
& (x+N) \odot(y+N)=\{z+N \mid z \in x \circ y\}
\end{aligned}
$$

we obtain that $(R / N, \oplus, \odot, \odot)$ is a composition hyperring, called the quotient composition hyperring related to the equivalence $\rho$.

Proof. First we prove that the hyperoperations $\oplus, \odot, \odot$ are well-defined.

Let $x+N=x_{1}+N$ and $y+N=y_{1}+N$, for $x, x_{1}, y, y_{1} \in R$. Set $L=(x+N) \oplus(y+N)=\{z+N \mid z \in x+y\}$ and $D=\left(x_{1}+N\right) \oplus\left(y_{1}+N\right)=$ $\left\{z+N \mid z \in x_{1}+y_{1}\right\}$. If $z \in x+y$, then $z+N \subseteq x+y+N=(x+N)+(y+N)=$ $\left(x_{1}+N\right)+\left(y_{1}+N\right)=x_{1}+y_{1}+N$. Since $z \in z+N \subseteq x_{1}+y_{1}+N$, there exists $z_{1} \in x_{1}+y_{1}$ and $n_{1} \in N$, such that $z \in z_{1}+n_{1}$. It follows that $z+N \subseteq z_{1}+n_{1}+N=z_{1}+N$. But $z \in z_{1}+n_{1}$, so $z_{1} \in z-n_{1}$ and then $z_{1}+N \subseteq z-n_{1}+N=z+N$. Thus, $z+N=z_{1}+N$, while $z_{1} \in x_{1}+y_{1}$. Therefore, $L \subseteq D$. Similarly one proves that $D \subseteq L$.

Now set $L=(x+N) \odot(y+N)=\{z+N \mid z \in x \cdot y\}$ and $D=\left(x_{1}+\right.$ $N) \odot\left(y_{1}+N\right)=\left\{z+N \mid z \in x_{1} \cdot y_{1}\right\}$. Let $z \in x \cdot y$. Since $x \in x_{1}+N$ and $y \in y_{1}+N$, there exist $n_{1}, n_{2} \in N$ such that $z \in\left(x_{1}+n_{1}\right) \cdot\left(y_{1}+n_{2}\right)=$ $x_{1} \cdot y_{1}+n_{1} \cdot y_{1}+x_{1} \cdot n_{2}+n_{1} \cdot n_{2} \subseteq x_{1} \cdot y_{1}+N$. Thereby, there exist $z_{1} \in x_{1} \cdot y_{1}$ and $n_{1} \in N$ such that $z \in z_{1}+n_{1}$. It implies that $z+N=z_{1}+N$, i.e. $z+N \in D$. So, $L \subseteq D$. The converse inclusion can be similarly proved.

Suppose now $L=(x+N) \odot(y+N)=\{z+N \mid z \in x \circ y\}$ and $D=$ $\left(x_{1}+N\right) \odot\left(y_{1}+N\right)=\left\{z+N \mid z \in x_{1} \circ y_{1}\right\}$. Let $z \in x \circ y$. Because $y \in y_{1}+N$, we can write $y \in y_{1}+n_{1}$, for some $n_{1} \in N$. It follows that $n_{1} \in y-y_{1}$, that is $y-y_{1} \cap N \neq \emptyset$. So $x \circ y-x \circ y_{1} \subseteq N$, i.e. $x \circ y \subseteq x \circ y_{1}+N$. Since $x \in x_{1}+N$, there exists $n_{2} \in N$ such that $x \in x_{1}+n_{2}$ and then $x \circ y \subseteq\left(x_{1}+n_{2}\right) \circ y_{1}+N=x_{1} \circ y_{1}+n_{2} \circ y_{1}+N \subseteq x_{1} \circ y_{1}+N$. Thereby, if $z \in x \circ y$, then there exists $z_{1} \in x_{1} \circ y_{1}$ such that $z \in z_{1}+N$ and thus $z+N=z_{1}+N$, which means that $L \subseteq D$ and similarly $D \subseteq L$.

Finally, it is easy to verify that $(R / N, \oplus, \odot, \odot)$ is a composition hyperring. We omit here the classical proof.

Definition 4.3. Let $R_{1}$ and $R_{2}$ be composition hyperrings. A mapping $f$ : $R_{1} \longrightarrow R_{2}$ is called a strong homomorphism if the following conditions are satisfied, for all $x, y \in R_{1}$ :

1. $f(x+y)=f(x)+f(y)$

2. $f(x \cdot y)=f(x) \cdot f(y)$ 
3. $f(x \circ y)=f(x) \circ f(y)$

4. $f(0)=0$.

A strong homomorphism $f$ is an isomorphism if $f$ is one to one and onto. We write $R_{1} \cong R_{2}$ if $R_{1}$ is isomorphic with $R_{2}$.

Notice that, if $f$ is a strong homomorphism from $R_{1}$ into $R_{2}$, then, for all $x \in R_{1}$, it holds $f(-x)=-f(x)$. Indeed, since $0 \in x-x$, it follows that $0=f(0) \in f(x)+f(-x)$, so $f(-x)=-f(x)$.

If $f$ is a strong homomorphism from $R_{1}$ into $R_{2}$, then the kernel of $f$ is the set $\operatorname{Kerf}=\left\{x \in R_{1} \mid f(x)=0\right\}$. Obviously, Kerf is a hyperideal of $\left(R_{1},+, \cdot\right)$, but generally it is not a composition hyperideal.

In the following, we will state and prove the isomorphism theorems for composition hyperrings. Note that, for the first theorem we need Kerf to be a composition hyperideal.

Theorem 4.4. Let $R_{1}$ and $R_{2}$ be composition hyperrings. If $f$ is a strong homomorphism from $R_{1}$ into $R_{2}$ with the kernel $K$ such that $K$ is a composition hyperideal of $R_{1}$, then $R_{1} / K \cong I m f$.

Proof. Define $\phi: R_{1} / K \longrightarrow \operatorname{Imf}$ by $\phi(x+K)=f(x)$, for all $x \in R_{1}$. First we prove that $\phi$ is well-defined. Suppose that $x+K=y+K$. Then, there exists $z \in K$ such that $x \in y+z$. It follows that $z \in(-y+x) \cap K$, that is $0=f(z) \in f(x)-f(y)$. Thus, $f(x)=f(y)$. Obviously, $f$ is onto. It remains to show that $\phi$ is one to one. Suppose $\phi(x+K)=\phi(y+K)$. Then $f(x)=f(y)$, which means that $0 \in f(x-y)$. Thus, there exists $z \in x-y$ such that $z \in K=K \operatorname{erf}$ and so, $x \in z+y \subseteq K+y$ which implies that $x+K=y+K$. Thereby $\phi$ is a bijection.

Moreover, $\phi$ is a strong homomorphism, because

$$
\begin{aligned}
\phi((x+K) \oplus(y+K)) & =\phi(\{z+K \mid z \in x+y\})=\{f(z) \mid z \in x+y\}= \\
& =f(x+y)=f(x)+f(y)=\phi(x+K)+\phi(y+K) .
\end{aligned}
$$

Similarly,

$$
\begin{array}{ll}
\phi((x+K) \odot(y+K)) & =\phi(x+K) \cdot \phi(y+K), \\
\phi((x+K) \odot(y+K)) & =\phi(x+K) \circ \phi(y+K)
\end{array}
$$

and $\phi(K)=f(0)=0$.

Theorem 4.5. If $A$ and $B$ are composition hyperideals of a composition hyperring $R$, then $A /(A \cap B) \cong(A+B) / B$. 
Proof. Clearly $A \cap B$ is a composition hyperideal of $A$ (as the intersection between composition hyperideals) and $A+B$ is a subhyperring of $(R,+, \cdot)$. If $x, y \in A+B$, then there exist $a, a_{1} \in A$ and $b, b_{1} \in B$ such that $x \in a+b$ and $y \in a_{1}+b_{1}$, and therefore $x \circ y \subseteq(a+b) \circ\left(a_{1}+b_{1}\right)=\bigcup_{s \in a_{1}+b_{1}}(a+b) \circ s=$ $\bigcup_{s \in a_{1}+b_{1}}(a \circ s+b \circ s) \subseteq \bigcup_{a^{\prime} \in A, b^{\prime} \in B} a^{\prime}+b^{\prime}=A+B$. Thus $A+B$ is a composition hyperring and, since $B$ is a composition hyperideal of $A+B$, it follows that $(A+B) / B$ is well defined.

Let us take $f: A \longrightarrow(A+B) / B$ by $f(a)=a+B$. It is easy to verify that $f$ is a strong homomorphism.

We prove that $f$ is onto. Let $y+B \in(A+B) / B$, with $y \in a+b$, for some $a \in A$ and $b \in B$. Then $a \in y-b$, that is $a \in y+B$. Thus $a+B=y+B$, so $f(a)=y+B$.

Besides, for any $a \in A$, it holds:

$$
a \in \operatorname{Kerf} \Longleftrightarrow f(a)=B \Longleftrightarrow a+B=B \Longleftrightarrow a \in A \cap B .
$$

Thereby $\operatorname{Kerf}=A \cap B$, and by Theorem 4.4, we get the isomorphism $A /(A \cap$ $B) \cong(A+B) / B$.

Theorem 4.6. If $A$ and $B$ are composition hyperideals of a composition hyperring $R$ such that $A \subseteq B$, then $B / A$ is a composition hyperideal of $R / A$ and $(R / A) /(B / A) \cong R / B$.

Proof. As in the previous two theorems, one can verify that $B / A$ is a composition hyperideal of $R / A$ and that the application $f: R / A \longrightarrow R / B$, defined by $f(x+A)=x+B$, is a strong homomorphism of $R / A$ onto $R / B$ with $\operatorname{Kerf}=B / A$.

\section{Conclusions and future work}

The notion of hyperring is a natural generalization of that of ring, many properties of rings have been transferred to the case of hyperrings. This paper is a contribution to the development of the theoretical background of hyperrings starting from rings. The notion of composition ring introduced in 1962 [1] has been extended to that of composition hyperring, i.e. a hyperring $(R,+, \cdot, \circ)$ with a new hyperoperation $\circ$, called composition, which is associative and distributive with respect to the addition and multiplication of the hyperring. It is shown that the composition structure of $R$ can be determined by a certain class of multiendomorphisms of $R$. The three isomorphism theorems have been proved for this class of hyperstructures.

This research could be continued further, for instance the theory of hyperideals (maximal or prime hyperideals) could be developed in this context, or to study the composition near-hyperrings, starting from near-rings. 
Acknowledgements. The first author was partially supported by "Agencija za raziskovalno dejavnost Republike Slovenije, program $P 1-0285$ ".

\section{References}

[1] I. Adler, Composition rings, Duke Math. J., 29 (1962), 607-623.

[2] H. Babaei, M. Jafarpour, S.Sh. Mousavi, R-parts in hyperrings, Iran. J. Math. Sci. Inform., 7(2012), no.1, 59-71.

[3] J. Chvalina, S. Hoskova-Mayerova, A.D. Nezhad, General actions of hyperstructures and some applications, An. Ştiinţ. Univ. Ovidius Constanţa Ser. Mat., 21(2013), no.1, 59-82.

[4] A. Connes, C. Consani, The hyperring of adele classes, J. Number Theory, 131(2011), no.2, 159-194.

[5] B. Davvaz, A. Salasi, A realization of hyperrings, Comm. Algebra, 34(2006), 4389-4400.

[6] B. Davvaz, V. Leoreanu-Fotea, Hyperring theory and applications, International Accademic Press, Palm Harbor, U.S.A., 2007.

[7] S. Jančić-Rašović, About the hyperring of polynomials, Ital. J. Pure Appl. Math., 21(2007), 223-234.

[8] S. Jančić-Rašović, On a class of Chinese Hyperrings, Ital. J. Pure Appl. Math., 28(2011), 245-256.

[9] M. Krasner, Approximations des corps values complets de characteristique $p \neq 0$ par ceux de characteristique 0, Colloque d'Algebre Superieure, CBRM, Bruxelles, 1956.

[10] M. Krasner, A class of hyperrings and hyperfields, Int. J. Math. Math. Sci., 6(1983), no.2, 307-312.

[11] Ch.G. Massouros, On the theory of hyperrings and hyperfields, Algebra i Logika, 24(1985), 728-742.

[12] G. G. Massouros, Fortified Join Hypergroups and Join Hyperrings, An. Ştiinţ. Univ. Al. I. Cuza Iaşi. Mat. (N.S.), 41(1995), 37-44.

[13] S. Mirvakili, B. Davvaz, Relations on Krasner $(m, n)$-hyperrings, European J. Combin., 31(2010), 790-802.

[14] J. Mittas, Hyperanneaux canoniques, Math. Balkanica, 2(1972), 165-179. 
[15] J. Mittas, Sur les hyperanneaux et les hypercorps, Math. Balkanica, 3(1973), 368-382.

[16] A. Nakassis, Recent results in hyperring and hyperfield theory, Int. J. Math. Math. Sci., 11(1988), 209-220.

[17] W. Phanthawimol, Y. Punkla, K. Kwakpatoon, Y. Kemprasit, On homomorphisms of Krasner hyperrings, An. Ştiinţ. Univ. Al. I. Cuza Iaşi. Mat. (N.S.), 57(2011), 239-246.

[18] S. Spartalis, A class of hyperrings, Riv. Mat. Pura Appl., 4(1989), 55-64.

[19] S. Spartalis, $(H, R)$-hyperrings, Algebraic hyperstructures and applications (Xanthi, 1990), 187195, World Sci. Publ., Teaneck, NJ, 1991.

[20] D. Stratigopoulos, Hyperanneaux non commutatifs: Le radical d'un hyperanneau, somme sous-directe des hyperanneaux artiniens et theorie des elements idempotents, C.R. Acad. Sci. Paris, 269(1969), 627-629.

[21] O. Viro, On basic concepts of tropical geometry, Tr. Mat. Inst. Steklova, 273(2011), Sovremennye Problemy Matematiki, 271-303.

[22] T. Vougiouklis, The fundamental relation in hyperrings. The general hyperfields, Algebraic hyperstructures and applications (Xanthi, 1990), 203211, World Sci. Publ., Teaneck, NJ, 1991.

Irina CRISTEA,

Centre for Systems and Information Technologies,

University of Nova Gorica

SI-5000, Vipavska 13, Nova Gorica, Slovenia.

Email: irinacri@yahoo.co.uk

Sanja JANČIĆ-RAŠOVIĆ,

Department of Mathematics, Faculty of Natural Science and Mathematics,

University of Montenegro,

Dzordza Vasingtona bb, 81000 Podgorica, Montenegro.

Email: sabu@t-com.me 